\title{
Ultrasonic Trilateration Methods for Low-Cost Indoor Positioning
}

\author{
Ismaila Adeniyi Kamil ${ }^{*} \quad$ Taiwo Damilola Fasae \\ Department of Electrical \& Electronic Engineering, University of Ibadan, Ibadan, Nigeria
}

\begin{abstract}
Trilateration is a process where distances to known control points or beacons can be used as inputs to algorithms to calculate an object's position. Most of the common trilateration methods known are fast and reliable, and based on estimating one or more location-dependent signal parameters like Times-of-arrival (TOA), Angle of Arrival (AOA) or Received Signal Strength (RSS). However, costly hardware is needed for such approaches to run reliably, and are too computationally complex both in speed and memory for low-cost hardware. The demand for low-cost hardware has greatly increased in recent years mostly due to the rapid emergence of internet of things, that promises to bring connectivity to just about everything. As such, leveraging on cheap hardware is an important step to take to achieve accurate indoor positioning. Because of convenience in generation and transmission, ultrasound is widely and commonly used electronically for distance measurement. The use of ultrasound with cheap hardware for accurate indoor positioning is discussed in this paper. Three localization methods that are reliable and fast enough for cheap hardware are analyzed with emphasis on the speed and memory demands. Experimental results show that the Maximum Likelihood estimation approach is the most accurate.
\end{abstract}

Keywords: Time of arrival; Trilateration; Ultrasound; Localization; Indoor positioning.

DOI: $10.7176 / \mathrm{ISDE} / 10-5-04$

Publication date: June $30^{\text {th }} 2019$

\section{Introduction}

Location awareness is one of the most sought-after services in the technical world today. Location-based services (LBS) takes in a user's position and provide context-aware information to the user or perform a more relevant task. Due to the importance of LBS, new areas of research and technological focus have been inspired, with tremendous potential in personal navigation and security, health-care, disability aids, etc. For example, a mobile device could be made capable of providing a user with directions within a large building in real-time; the ability to print to the nearest printer or access the nearest resource irrespective of your physical location; or the ability to receive from your personal digital assistant (PDA), audible directions to your seat in an airplane.

Outdoor LBS have become very popular today, with users getting even real-time location-based suggestions and assistance on PDAs and mobile phones. Despite the role GPS has played in the success of outdoor LBS, providing accuracies within meters of user's location, it is not so much as attractive in indoor applications: higher accuracy is required for indoor environments but GPS signals (as electromagnetics) suffer from fading and attenuation of walls, windows, and roofs. These factors make indoor positioning very challenging.

Attention has hence shifted to other propagation methods such as Radio Frequency (RF) signals used in GSM, Wi-Fi and Bluetooth and have quite been successful in achieving better indoor accuracy than the GPS, the Active Badge system (Want, et al., 1992) for example pin-points the room (and floor) an object carrying an RFID badge is located. However, specialized hardware is needed to obtain some significant level of accuracy. Ultrasound easily becomes a choice, because of its frequency range, between 20 and $22 \mathrm{KHz}$, which is high enough to be inaudible by humans but still low enough to be generated by cheap hardware. And because ultrasound is a type of sound, its speed, about $340 \mathrm{~m} / \mathrm{s}$, is much slower than RF signals, so it's much longer time of flight enables us to measure centimeter-level distances.

The afore-mentioned properties of ultrasound make the adoption for high accuracy-demanding positioning systems popular today. The Active Bat System (Ward, et al., 1997) and the Cricket System (Priyantha, et al., 2000) are well-known systems that are based on the Times-of-Arrival (TOA) of ultrasound travel to estimate the device's position (Holm, 2012) (Sanchez, et al., 2012). In each of the systems, the TOA, as a location-dependent parameter, is used in a first step to estimate the distances from the emitter to the receivers. Then in a second step, the collection of estimated ranges is used to determine the estimate of the emitter's location. This two-stage procedure is the classic approach to source localization.

Despite the success of the afore-mentioned ultrasound-based systems, each system is heavily dependent on using a PC for accomplishing at least one stage of the localization process. Using a PC or external support system would most often improve the positioning accuracy. However, implementing such PC-dependent setup is too costly for small applications. In (Ahmad, et al., 2016), low-cost FPGAs are introduced. By accomplishing location estimates neither using a PC nor any external support system, the cost of setting up is brought to a low value, although the system could depend on an external PC for improved accuracy.

The second stage of the localization process is important in achieving the desired level of accuracy, and often 
demand the most attention. Distance and range measurements (outcome of the first-stage in the process) would now serve as inputs into the second stage. This important step involves methods such as trilateration, where distances to known control points or beacons can be used as inputs to algorithms to calculate the object's position. We discuss three trilateration methods and prove each to be suitable for low-cost hardware. Despite the myriads of robust solutions to trilateration, a lot of which are designed without hardware limitations in mind, only a few are suitable to run with limited memory and on cheap hardware. So, if low-cost indoor positioning would be achieved, we must explore these limited possibilities

\section{Trilateration Methods}

As discussed in the introduction, by using trilateration it is possible to calculate one's position based on the distance to several other(control) points with known positions (Bossler, et al., 2010) (Ghilani \& Wolf., 2006). To find one's position in 2 dimensions the number of required known points is 3 ; for position in 3 dimensions the number of known points is 4 . The time it takes a signal to travel between the object, with unknown position, to each control point can be directly converted to distance. This is the TOA (Time of Arrival) approach, where both the time the signal was sent and the time it was received are required to get the time of flight. However, because synchronization between the sender and the receiver is necessary, TOA requires that times from the two separate systems have to be synchronized. This is a rather difficult problem, even more for cheap electronics to manage. The Time Difference of Arrival (TDOA) approach solves this problem by requiring only the differences in TOA at each receiver.

\subsection{Maximum Likelihood Estimation}

The Maximum Likelihood Estimation (MLE) method estimates, by finding the parameter values that maximize the likelihood of making the observation given the parameters, for a given statistical model.

To apply this method, the likelihood function, $\operatorname{lik}(\theta)=f\left(x_{1}, x_{2}, \ldots, x_{m} \mid \theta\right)$, of the observed data has to be specified, where $\operatorname{lik}(\theta)$ is the probability of observing the given data as a function of $\theta$. If the $x_{i}$ are independent and identically distributed, then $\operatorname{lik}(\theta)=\prod_{i=1}^{m} f\left(x_{i} \mid \theta\right)$ (Kay, 1993). In our usage here, $\theta$ is deterministic and represents the exact TDOA from sensor $i$ to the reference sensor.

Maximizing the likelihood function is equivalent to maximizing the log of the likelihood function (Bossler, et al., 2010), thus

$$
l(\theta)=\sum_{i=1}^{m} \log \left(f\left(x_{i} \mid \theta\right)\right)
$$

Since our signal model is normally distributed with mean, $\mu=\theta$ and variance, $\sigma$. Then,

and $\log$ likelihood function (Bossler, et al., 2010):

$$
\operatorname{lik}(\theta)=P(Y \mid \theta)=\prod_{j=1}^{T} \prod_{i=2}^{m} e^{\frac{-\left(Y_{i, j}-\theta_{i}\right)^{2}}{2 \sigma^{2}}}
$$

$$
l(\theta)=\frac{-1}{2 \sigma^{2}} \sum_{j=1}^{T} \sum_{i=2}^{m}\left(Y_{i, j}-\theta_{i}\right)^{2}
$$

This amounts to minimizing the sum of squared errors, $\left(Y_{i, j}-\theta_{i}\right)^{2}$ for our deterministic, unknown parameter, $\theta$. Implementing this algorithm involves searching every integer coordinate point in a given range to determine which $(x, y)$ point result in the smallest mean-squared-error (MSE) for the $m-1$ TDOA distances for T number of measurements. This means converting each of the $(x, y)$ coordinates in our search space into exact TDOA measurements; finding the differences from our estimated TDOA measurements; squaring and averaging the T number of measurements across the $m-1$ TDOA distances.

This implies that for each $(x, y)$ point in space, the mean-squared-error (MSE)

$$
M S E=\frac{1}{m-1} * \frac{1}{T} * \sum_{j=1}^{T} \sum_{i=2}^{m}\left(Y_{i, j}-\theta_{i}\right)^{2}
$$

The point with the smallest MSE is the estimate of the desired position.

\subsection{Simple Least Squares Approach}

The Least Squares approach is one of the most popular statistical approaches to TDOA trilateration (Jin, et al., 2018). It is a standard approach in regression analysis to approximate the solution of overdetermined systems, especially equations with more equations than unknowns. The Least Squares approach has the ability to tolerate errors in measurements; with more measurements provided, less is the impact from a single erroneous measurement. In this work, the simple least squares approach refers to the least squares approach in its basic simplicity.

From our TDOA problem, the resulting equations are arranged in matrix form,

$$
A \boldsymbol{x}=B
$$

where $\boldsymbol{x}$ is a column vector containing all the unknowns: 


$$
\boldsymbol{x}=\left(\begin{array}{c}
x \\
y \\
z \\
r_{1}
\end{array}\right)
$$

$A$ is a $(N-1) \times 4$ matrix and $B$ is a column vector of length $N-1$ given by:

$$
\begin{gathered}
A=\left(\begin{array}{cccc}
2\left(x_{2}-x_{1}\right) & 2\left(y_{2}-y_{1}\right) & 2\left(z_{2}-x_{1}\right) & 2 \Delta r_{21} \\
2\left(x_{3}-x_{1}\right) & 2\left(y_{3}-y_{1}\right) & 2\left(z_{3}-x_{1}\right) & 2 \Delta r_{31} \\
\vdots & \vdots & \vdots & \vdots \\
2\left(x_{N}-x_{1}\right) & 2\left(y_{N}-y_{1}\right) & 2\left(z_{N}-x_{1}\right) & 2 \Delta r_{N 1}
\end{array}\right) \\
B=\left(\begin{array}{c}
x_{2}^{2}+y_{2}^{2}+z_{2}^{2}-x_{1}^{2}-y_{1}^{2}-z_{1}^{2}-2 \Delta r_{21}^{2} \\
x_{3}^{2}+y_{3}^{2}+z_{3}^{2}-x_{1}^{2}-y_{1}^{2}-z_{1}^{2}-2 \Delta r_{31}^{2} \\
\vdots \\
x_{N}^{2}+y_{N}^{2}+z_{N}^{2}-x_{1}^{2}-y_{1}^{2}-z_{1}^{2}-2 \Delta r_{N 1}^{2}
\end{array}\right)
\end{gathered}
$$

These matrices depend on beacon positioning coordinates $x_{k}, y_{k}, z_{k}$ and measured differences of range $\Delta r_{k 1}$. Using the Least Squares formulation,

$$
X=\left(A^{T} \cdot A\right)^{-1} \cdot A^{T} \cdot B
$$

\subsection{Lok8 Least Squares Approach}

This approach was developed to introduce iteration to improve approximations to the least squares approach (Filonenko, et al., 2012) (Filonenko, et al., 2013).

Given:

where in our case:

$$
{ }_{m} A_{n}+{ }_{n} X_{1}={ }_{m} L_{1}+{ }_{m} V_{1},
$$

- $m=3, n=2$

- ${ }_{m} A_{n}$ contains the coefficients of the unknowns $(x, y)$

- ${ }_{n} X_{1}$ contains the corrections to be applied to the initial estimates for the unknowns $(d x, d y)$

- $\quad{ }_{m} L_{1}$ contains the measurements $\left(r_{21}, r_{31}, r_{41}\right)$

- ${ }_{m} V_{1}$ contains the residuals (one for each measurement)

Solving for $X$ gives the solution:

where:

$$
X=\left(A^{T} \cdot A\right)^{-1} \cdot A^{T} \cdot L
$$

$$
\begin{gathered}
A=\left(\begin{array}{lll}
\frac{\left(x-x_{2}\right)}{r_{1}+\Delta r_{21}}-\frac{\left(x-x_{1}\right)}{r_{1}} & \frac{\left(y-y_{2}\right)}{r_{1}+\Delta r_{21}}-\frac{\left(y-y_{1}\right)}{r_{1}} \\
\frac{\left(x-x_{3}\right)}{r_{1}+\Delta r_{31}}-\frac{\left(x-x_{1}\right)}{r_{1}} & \frac{\left(y-y_{31}\right)}{r_{1}+\Delta r_{31}}-\frac{\left(y-y_{1}\right)}{r_{1}} \\
\frac{\left(x-x_{4}\right)}{r_{1}+\Delta r_{41}}-\frac{\left(x-x_{1}\right)}{r_{1}} & \frac{\left(y-y_{4}\right)}{r_{1}+\Delta r_{41}}-\frac{\left(y-y_{1}\right)}{r_{1}}
\end{array}\right) \\
X=\left(\begin{array}{l}
d x \\
d y
\end{array}\right) ; L=\left(\begin{array}{l}
\Delta r_{21}-\Delta r_{21}^{\prime} \\
\Delta r_{31}-\Delta r_{31}^{\prime} \\
\Delta r_{41}-\Delta r_{41}^{\prime}
\end{array}\right) ; V=\left(\begin{array}{l}
v_{m 2} \\
v_{m 3} \\
v_{m 4}
\end{array}\right)
\end{gathered}
$$

$X$ contains the corrections to be applied to the original estimates for $(x, y)$. These new $(x, y)$ coordinates are then used to recalculate updated distances for $\left(r_{1}, \Delta r_{21}^{\prime}, \Delta r_{31}^{\prime}, \Delta r_{41}^{\prime}\right)$. The process is repeated until the coordinates of $(x, y)$ don't change significantly.

The initial estimates for $x$ and $(x, y)$ can be set to the average of $x$ and $y$ coordinates of the four receivers, and $r_{1}$ is then calculated using Pythagoras theorem at $x$ and $y$ :

$$
\begin{gathered}
r_{1}=\sqrt{\left(x-x_{1}\right)^{2}+\left(y-y_{1}\right)^{2}} \\
\Delta r_{21}^{\prime}=\sqrt{\left(x-x_{2}\right)^{2}+\left(y-y_{2}\right)^{2}}-r_{1} \\
\Delta r_{31}^{\prime}=\sqrt{\left(x-x_{3}\right)^{2}+\left(y-y_{3}\right)^{2}}-r_{1} \\
\Delta r_{41}^{\prime}=\sqrt{\left(x-x_{4}\right)^{2}+\left(y-y_{4}\right)^{2}}-r_{1}
\end{gathered}
$$

\section{Experimental Setup}

Emphasis in this work is on the trilateration phase of the localization process, and no specific setup is proposed, but a simple low-cost setup is described. As shown in Figure 1, there is an active ultrasonic transmitter on the mobile unit and four passive receiver units with known fixed positions. In this work, tests were carried out on the three trilateration methods, each of which required TDOA measurements. The environment used is a $200 \mathrm{~cm}$ by 
$200 \mathrm{~cm}$ square area partitioned into $10 \mathrm{~cm}$ by $10 \mathrm{~cm}$ grids, with sensors placed at the four corners of the square area. For time synchronization, RF transceivers are installed on the mobile unit and fusion center depending on communication preferences. In this setup, the mobile unit begins the positioning step by emitting RF messages that carry information about the ultrasonic signals being sent. The fusion center consequently uses this synchrony information to derive the receivers' time of flight for position calculation, which is displayed or sent to the mobile unit for secondary purposes e.g. navigation, location-awareness. To visualize the accuracies in the system, meansquared-errors of 20 measurements per grid, between the known actual location and the estimated location are calculated and plotted against the grid points in 3-D surface plots. In addition, ways to improve accuracy in each trilateration method are discussed.

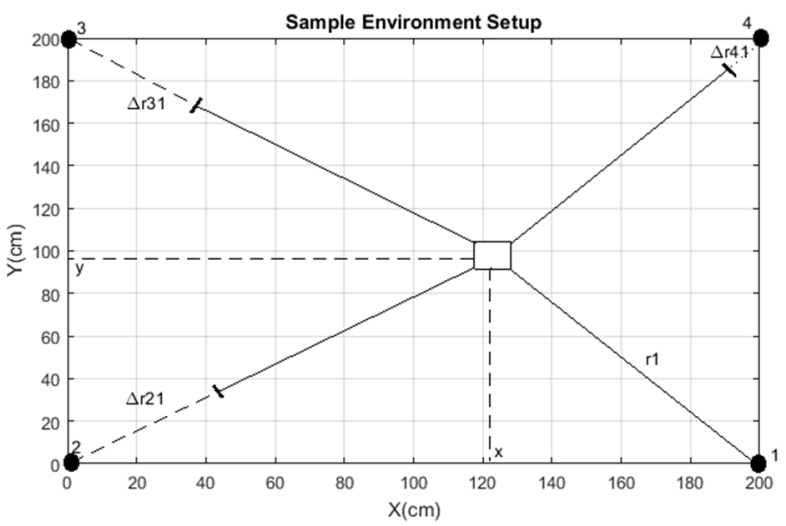

Figure 1. A sample indoor environment

\section{Results and Discussion}

Using the maximum likelihood estimation method, the mean square errors were as low as $3 \mathrm{~cm}$ as shown in Figure 2. MSE was lower than $10 \mathrm{~cm}$ in majority of the parts of the environment. While this method seems to be a viable approach, it takes considerable time to achieve this height of accuracy, since the entire search space in the domain must be scanned for the data points that result in the lowest MSE for a particular measurement. However, the time taken is dependent on the data points in the domain, so in domestic applications where there are few data points and lesser emphasis on granularity, this method is considerably fast enough. Furthermore, the MLE is not too demanding in terms of memory, since calculations - sums and squares - can be performed per data point and results are stored in a global memory location.



Figure 2. MSE Plot for the Maximum Likelihood Estimation Approach

Using the least squares method, as seen in Figure 3, MSE was quite as high as $40 \mathrm{~cm}$ in majority of the parts of the environment. This approach is most suited for fast (short) times and low accuracy demands, since the time is only spent on matrix calculations, also the measurement errors have significant impact in the outcome of the calculations, as can be seen in the shades of yellow in the Figure 3. 


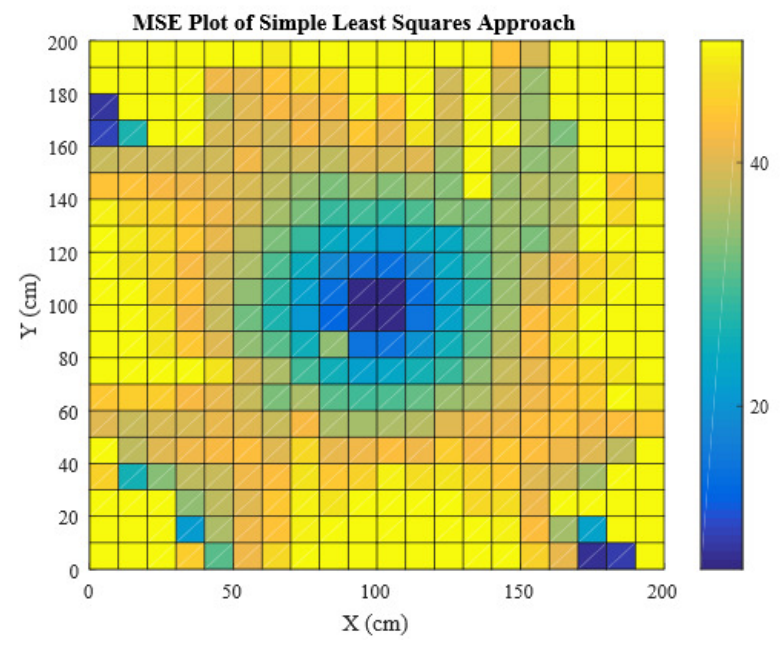

Figure 3. MSE Plot for the Traditional Least Squares Approach

In the Lok8 least squares method, from Figure 4, MSE was less than $20 \mathrm{~cm}$ in majority of the parts of the environment. As an improvement on the least squares approach, the iteration steps are shown to significantly reduce the impact of measurement errors in the result. Although the iteration steps may seem to require much longer time, most of the data points had iteration steps of 3 or 4 in number. Hence, while leveraging on the speed of least squares approach, the Lok8 approach reduces significantly the errors in the trilateration process.



Figure 4. MSE Plot for the Lok8 Least Squares Approach

\section{Conclusion}

Positioning is becoming an everyday need. As such, localization devices must be simple and cheap enough to encourage adoption. In this work, we have compared three trilateration methods that are efficient and costeffective for domestic or needs. The Maximum Likelihood estimation approach is very accurate, but needs more information about the environment and more time to determine the point with the least minimum square error. The least squares approach is fast enough, but easily prone to measurement errors. The Lok8 least squares approach improves accuracy by introducing iteration. The choice of any of these three techniques depends on the application it is intended for.

\section{References}

Ahmad, N. et al., (2016). Determining the ultrasound time of response using FPGA-based electronics read-out. 2016 IEEE EMBS Conference on Biomedical Engineering and Sciences (IECBES)

Bossler, J. D., Campbell, J. B., McMaster, R. B. \& Rizos, C., (2010). Manual of Geospatial Science and Technology. 2nd ed. s.1.:CRC Press.

Filonenko, V., Cullen ,. C. \& Carswell, J. D., (2013). Indoor Positioning for Smartphones Using Asynchronous Ultrasound Trilateration. ISPRS International Journal of Geo-Information, Volume 2, pp. 598-620.

Filonenko, V., Cullen, C. \& Carswell , J. D., (2012). Asynchronous Ultrasonic Trilateration for Indoor Positioning of Mobile Phones.. Dublin, Ireland, SpringerLink, pp. 33-46.

Ghilani, C. D. \& Wolf., P. R., (2006). Adjustment Computations: Spatial Data Analysis. s.1.:John Wiley \& Sons, 
Inc..

Holm, S., (2012). Ultrasound positioning based on time-of-flight and signal strength. Sydney, Australia, IEEE.

Jin, B., Xu , X. \& Zhang, T., (2018). Robust Time-Difference-of-Arrival (TDOA) Localization Using Weighted Least Squares with Cone Tangent Plane Constraint. Sensors, 18(3), pp. 1-16.

Kay, S. M., (1993). Fundamentals of Statistical Signal Processing: Estimation Theory. 1st ed. s.1.:Prentice hall.

Priyantha, N. B., Chakraborty, A. \& Balakrishnan., H., (2000). The Cricket Location-Support System. Boston, MA, s.n.

Sanchez, A. et al., (2012). Autonomous indoor ultrasonic positioning system based on alow-cost conditioning circuit. Measurement, Volume 45, pp. 276-283.

Sorbelli, F. B., Das, S. K., Pinotti, C. M. \& Silvestri, S., (2018). Precise Localization in Sparse Sensor Networks using a Drone with Directional Antennas. Varanasi, India, ACM New York, NY, USA.

Tuba, E., Tuba, M. \& Beko, M., (2018). Two Stage Wireless Sensor Node Localization Using Firefly Algorithm.. s.l., Springer, pp. 113-120.

Want, R., Hopper, A., Falcão, V. \& Gibbons, J., (1992). The active badge location system.. ACM Transactions on Information Systems, 10(1), pp. 91-102.

Ward, A., Jones, A. \& Hopper, A., (1997). A new location technique for the active office, 4(5) (1997) 42-27. IEEE Personal Communications, 4(5), pp. 27-42. 